\title{
ExoTen-Glove: A Force-Feedback Haptic Glove Based on Twisted String Actuation System
}

\author{
Mohssen Hosseini ${ }^{1}$ Ali Sengül ${ }^{2}$ Yudha Pane $^{1}$ Joris De Schutter ${ }^{1}$ and Herman Bruyninckx ${ }^{1,3}$
}

\begin{abstract}
This paper presents a novel lightweight and simple TSA-based (twisted string actuation) wearable haptic glove (ExoTen-Glove). This system is using two independent twisted string actuators with integrated force sensors and small-size DC motors. The proposed system can provide users force feedback during the execution of grasping virtual objects. The design of the TSA-based ExoTen-Glove, description of the TSA system, the controller and the preliminary experimental evaluation of the proposed system has been presented in this paper. This device has been evaluated by an experiment in virtual reality environment using HTC VIVE headset with 2 degrees of freedom grasping tasks, where the participants were squeezing a real spring with their thumb and index finger and compare it with a virtual spring stiffness. The results prove the applicability of the ExoTen-Glove for rehabilitation and haptic purposes.
\end{abstract}

Index Terms-Force feedback, Twisted String Actuation System, Wearable, Virtual Reality, Stiffness discrimination, Tendon Transmission System, Human Computer Interaction, Human haptic perception.

\section{INTRODUCTION}

Recently, there is an increasing interest in the development of robotics hand interfaces; an example of a popular device is hand exoskeleton. Such a device typically comes with two main purposes: to augment the fidelity of a teleoperated system and to be used for rehabilitation. For the teleoperation case, the hand exoskeleton is controlled to give a haptic feedback to the operator such that interaction with real or virtual objects becomes more realistic. On the rehabilitation case, the device guides the user hand's movement through a medically prescribed motion patterns, thus slowly recovering the user's motoric skills.

There have been many attempts in realizing such a device, with main applications on haptic interfaces for medical training [1], [2], for telemanipulation [3], [4] and for interaction with nano and micro scale phenomenon [5], [6]. In [7], a biometic hand device (BiomHED) was introduced to assist stroke patients in producing a variety of functional hand movements. The device was designed based on exotendons actuation with the capability of regenerating the distinct joint coordination patterns of human muscle-tendon units. A hand exoskeleton design based on underactuation was treated in [8], [9], with the emphasis on the analysis and optimization

\footnotetext{
*The support from VLAIO/Flanders Make Project FINROP_ICON is gratefully acknowledged.

${ }^{1}$ Robotics Research Group (a university core lab of Flanders Make), Department of Mechanical Engineering, KU LEUVEN, Belgium. (mohssen.hosseini, yudha.pane, joris.deschutter, herman.bruyninckx) @kuleuven. be

${ }^{2}$ Helbling Technik AG, Switzerland. ali.senguel@helbling.ch

${ }^{3}$ Department of Mechanical Engineering, TU/e, the Netherlands.
}

of the mechanism. A method to optimize the mechanism to provide proper finger movement and force is developed in [10]. In [11], [12], a five-fingered haptic interface with 6DOF arm and a 15-DOF hand that allow the user to interact with virtual objects, has been presented.

Virtual Reality (VR) technology has been explored for quite some time, with one of the earliest application in late 1950's. Today VR technologies are used in wide range of applications, simulating the real world dynamically and response in real time to the users. Integration of haptic feedback in VR can be traced back to NASA's Virtual Environment Workstation [13]. Nowadays, haptic interface is widely accepted as a means to augment VR. Most human interfaces use information of visual and auditory sensation. By adding force/tactile sensation, the users are expected to be able to perform more complex tasks while being physically aware of the objects they are manipulating. The different types of feedback which are commonly provided are force [14]-[17], tactile [18]-[20] or pressure feedback [21], [22].

Wearable systems with the force feedback allow the user to grasp, feel and manipulate the virtual objects in a natural way during interaction with the virtual environment. However, most of the wearable haptic devices use a substantial number of actuators, hence making them sometimes impractical for long term use. Designing a wearable force feedback device which is lightweight, less invasive, ergonomic while keeping the cost affordable is not a trivial problem. Using a new actuation method such as twisted string actuation (TSA) system would enable novel ways of wearable haptic devices to achieve the aforementioned design goal.

This paper presents a wearable haptic glove (ExoTenGlove) based on TSA system and exotendons. The TSA system [23], [24] is known for its lightweight and affordable properties. Thanks to the mechanical design, a TSA actuator can apply a relatively large force by relying on small DC motors. TSA actuators typically require DC motors with high-speed and low-torque, thus eliminating the need for

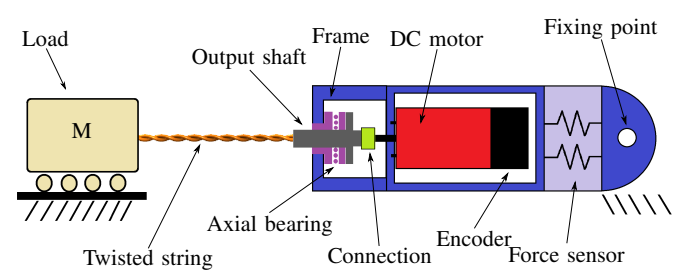

Fig. 1. Working principle of the twisted string actuation. 
gearboxes. TSA is commonly integrated with a powerful tendon-based driving system. Another advantageous characteristic of TSA is its slender structure which makes it particularly suitable to be used in highly integrated devices like robotic hands and exoskeletons. There are various applications of TSA in the literatures such as DEXMART hand [25], 1-DOF elbow exoskeleton [26] and a 6-DOF cabledriven haptic interface [27]. Therefore, the proposed ExoTenGlove is a promising approach to achieve a wearable haptic device that provides a realistic force feedback without any bulky mechanism such as gears.

This paper is organized as follows. Section II reviews some related works, whereas Section III describes the design of the overall system. In Section IV experimental evaluation of the device is reported, while in Section V comparison of the some existing device is presented. Finally, Section VI provides the conclusions and discusses future research activities.

\section{RELATED WORK}

There are few precursors works that are related to our research. The PHANToM [28] is one of the earliest haptic interface that provides a force feedback on the finger, allowing the users to feel the stiffness and textures of virtual objects. It is a 6-DOF externally grounded system with using three DC brushed motors and encoders. The disadvantage of PHANToM is its limited workspace and portability due to its grounded design. Furthermore, the variation of tasks that can be performed with the device is also limited since it only produces force feedback for one finger only. In order to grasp and manipulate virtual objects, using at least two fingers (thumb and index) is essential, e.g. a haptic glove, that allow users to grasp and feel the virtual objects in a more natural way while feeling their hardness. The CyberGrasp [29] is a commercial grounded force feedback system using cabledriven exoskeleton structure on the back of the hand which can provide an individual force, up to $12 N$, to each finger that can be combined with a separately available CyberGlove dataglove, providing joint angle information of the hand and fingers with a full wrist closure. The actuators are placed with a distance in the control box which results in a weight of only 450 grams. However, this introduces a trade-off as the system inherently has a high friction and backlashes. The Rutgers Master II-ND glove [30] is a haptic interface, developed at Rutgers University, designed for dexterous interactions with virtual environments that provides up to $16 \mathrm{~N}$ force feedback to each finger using pneumatic actuators. The drawback of this device is its lack of full finger closure capability due to the placement of the actuators in the palm. In [31], a mechanical exoskeleton system, called DEXMO, for motion capturing and force feedback for virtual reality is presented. This portable device weighs only $270 \mathrm{grams}$, affordable, and can track the user's motion and provide a force feedback. Choi et al. address the design, controller and performance analysis of Wolverine, a lightweight, low-cost and wearable haptic device to simulate the grasping of rigid objects in virtual environment. Their device is able to provide about $100 \mathrm{~N}$ force directly between the thumb and three

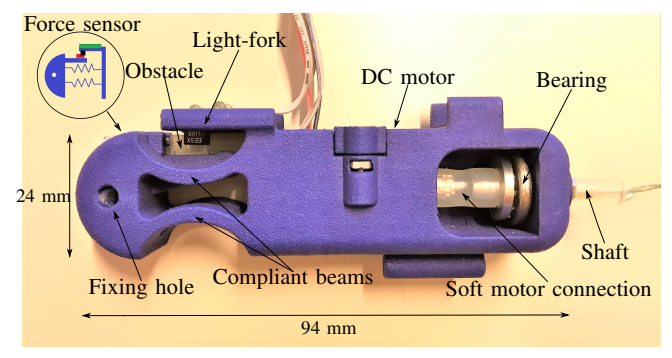

(a) Side view.

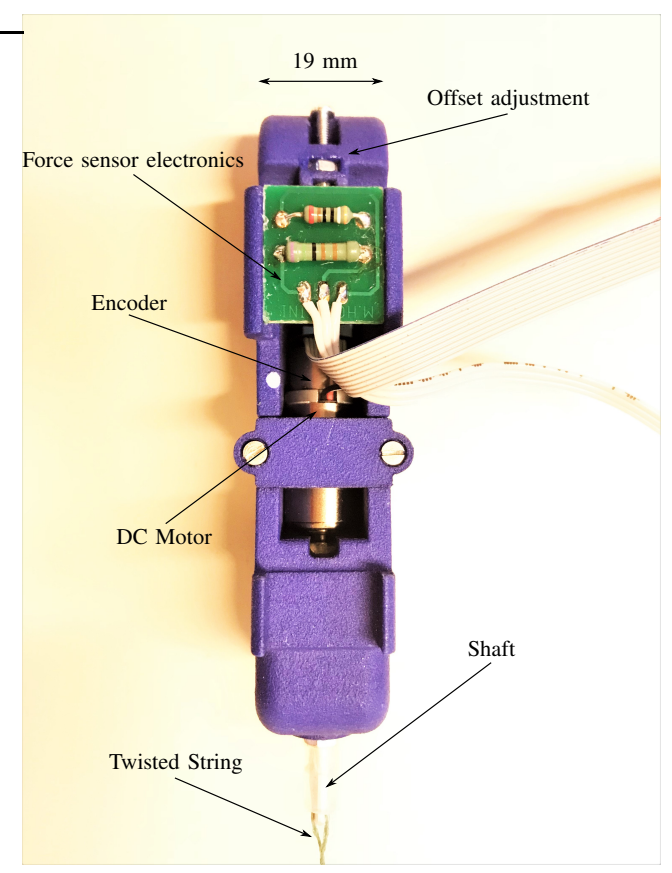

(b) Top view.

Fig. 2. Detailed view of the TSA module prototype.

fingers [32]. Another example of a wearable haptic interface is [33], (DESR) a light-weight force feedback device for the surgery simulation using Dielectric elastomer spring roll linear actuators with maximum force of 7.2N. However, due to the design, the device has a limited range of motion.

\section{SySTEM DESCRIPTION}

\section{A. TSA System}

The principle of the TSA is very simple: a pair of strings are connected from one end to a rotative DC motor and the other end to a linear moving element, i.e. the load to be actuated (see Fig. 1). The rotation imposed to the strings by the electrical motor reduces their length, generating a linear motion at the load side. The properties of the TSA system allow us to implement a low-cost, light-weight, and compact linear transmission system using small-size DC motors characterized by high speed and low torque. The mathematical model of the TSA system has been implemented in [23], [34]. 


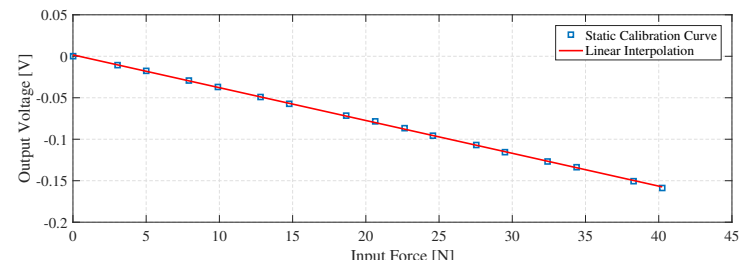

(a) Actuator no.1.

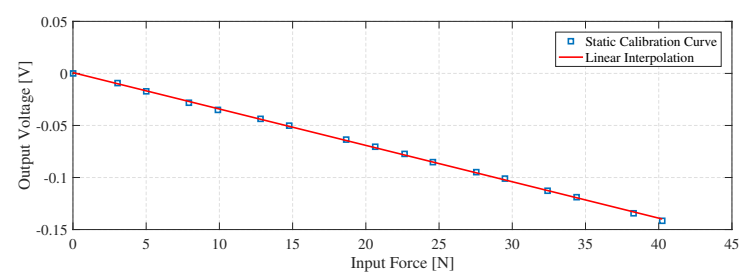

(b) Actuator no.2.

Fig. 3. Sensor calibration of both actuators.

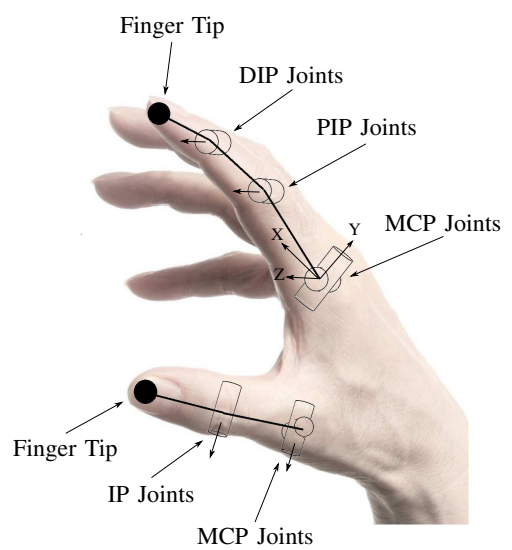

Fig. 4. Human finger structure.

\section{B. Force Sensor}

In order to measure and control the force applied by the twisted strings to the load directly, implementing a force sensor is mandatory [35], [36]. The sensor is based on a compliant frame deformation manufactured in Polyamide (PA 12) by Laser Sintering (SLS) and a commercial optoelectronic component called Light-fork (OMRON EE-SX1108), detecting frame deformation and convert it back to the applied force. This force sensor is taking advantages of a simple design, low-cost, the sensitivity and noise rejection.

\section{Actuation Module}

Figure 2 illustrates the detailed view of the TSA module with integrated force sensor that is used for implementation of the proposed ExoTen-Glove. The TSA module design has been described in details in [37], [38] thus here only a brief description of its main features is reported.

The actuator structure is composed of:

i) a frame hosting the DC motor (maxon DCX) and the encoder (maxon ENX), sensor electronic components,

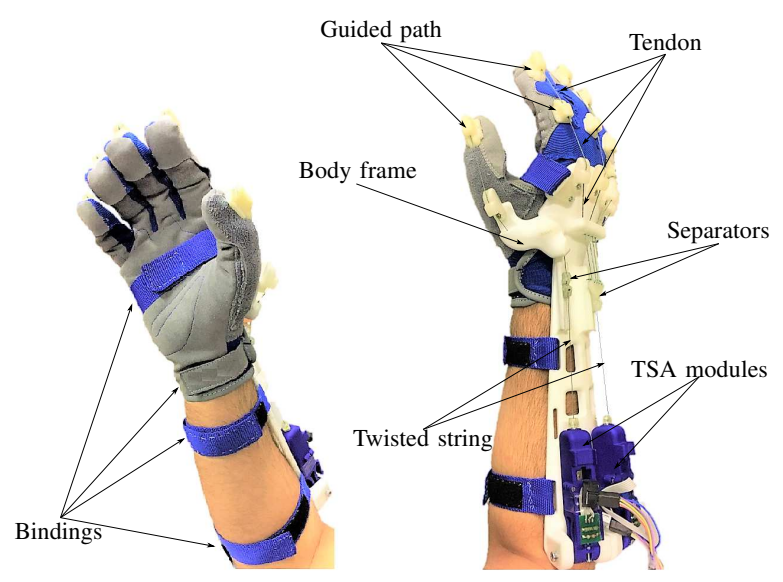

Fig. 5. Overview of the TSA based haptic glove.

with a dimension of $(94 \mathrm{~mm}$ length, $24 \mathrm{~mm}$ height, $19 \mathrm{~mm}$ width), the compliant frame is made of Plyamide (PA 12) manufactured by SLS;

ii) a fixing hole for connection to the supporting structure;

iii) an integrated force sensor;

iv) a double beam curved structure axial-symmetric; these beams function as a linear spring granting a certain compliance to the structure as well as the implementation of the force sensor;

v) a shaft supported by an axial bearing at the point of the twisted string connection to both reduce the friction and prevent the transmission force from damaging the motor;

vi) a silicon tube for coupling the motor and transmission shafts to provide ample flexibility in order to solve problems due to misalignment of the rotational axes of the motor and the transmission shaft;

vii) the string (Dyneema with a diameter of $0.16 \mathrm{~mm}$ ) itself connects the motor module with the linear moving element.

This particular structure allows the transmission force to be completely supported by the output shaft through a combined bearing while the motor is only used to transmit the necessary torque for driving the twisted string actuation to the output shaft.

The required length of transmission $p$ for the presented system can be calculated according to the kinetostatic model of the TSA [23]:

$$
p=\sqrt{L_{0}^{2}\left(1+\frac{F_{i}}{K}\right)^{2}-\theta^{2} r^{2}}
$$

where the $L_{0}$ is the unloaded length of the strings, $F_{i}$ the fiber tension, string stiffness $K$ (normalized with respect to the length unit), i.e. $r$ the string radius and $\theta$ is the motor angular position, respectively.

The actuator's force sensors has been calibrated and results have been presented in Figure 3. The total weight of an ExoTen-Glove actuator, including force sensor, DC motor, encoder and mechanical component is 40 grams. 

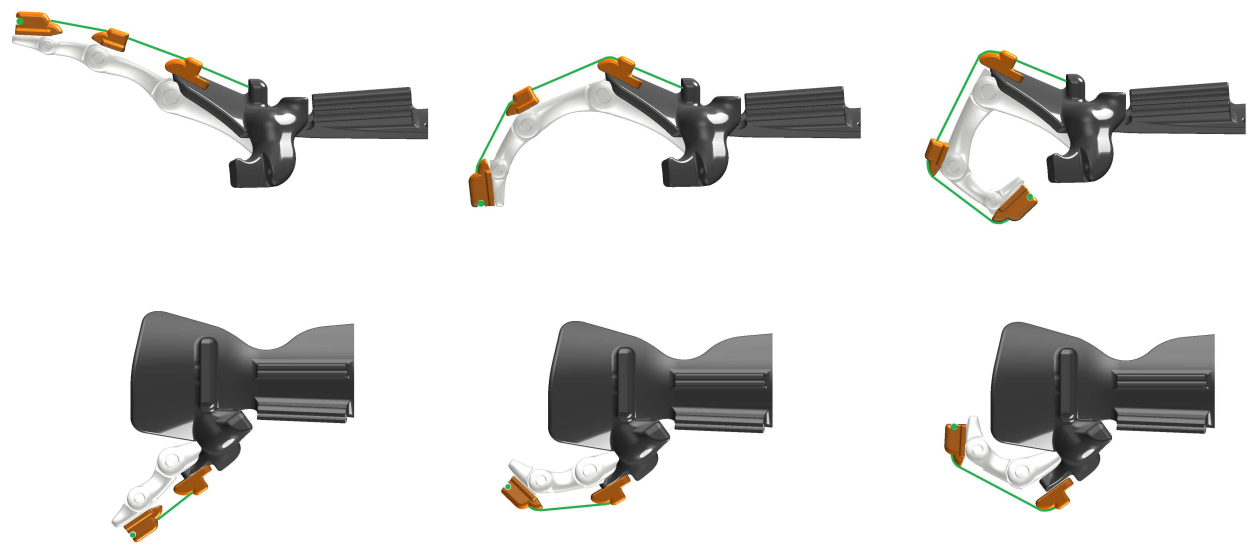

Fig. 6. Finger position and Tendon guideline.

\section{Fingers Structure}

The hand is comprised of five digits and they are called thumb, index, middle, ring and little finger. The four fingers from index to little finger have three phalanges: distal, intermediate and proximal phalanges and have three joints: Distal Interphalangeal (DIP), Proximal Interphalangeal (PIP) and Metacarpophalangeal (MCP) joints while the thumb comprised of two phalanges; distal and proximal phalanges and two joints: Interphalangeal (IP) and Metacarpophalangeal (MCP) joints (see Fig. 4).

\section{E. Design of the Haptic Glove}

As it can be seen in Figure 5, we presented a simple and light-weight TSA-based haptic glove (ExoTen-Glove) that has been designed to be worn comfortably without any external bulky mechanism to drive the fingers, with a full range of motion, by tendons using skeleton structure of human fingers. It composed by:

i) a rigid supporting frame with anatomical shape of forearm made of Polyamide (PA 12) by Laser Sintering (SLS);

ii) a comfortable commercial soft gloves; to be worn by user and mount the tendon guidelines;

iii) two independent TSA modules: to drive a group of fingers from index to pinky finger and the thumb separately for grasping tasks;

iv) tendons (Dyneema $0.26 \mathrm{~mm}$ ); to connect the fingers to the actuators guided through a path;

v) the bindings structure; to tighten the glove and the system on the user's hand, and on top of each fingers in order to prevent the slack;

vi) the guided path structure; made of semi-soft material 3D printed (EFLEX) that are attached on the glove; The path is designed with a curved shape to allow the tendons follow the finger movement with its anatomical shape and prevent the shear forces to the attachment points (see Fig. 6) which results in a full range of the hand motion;

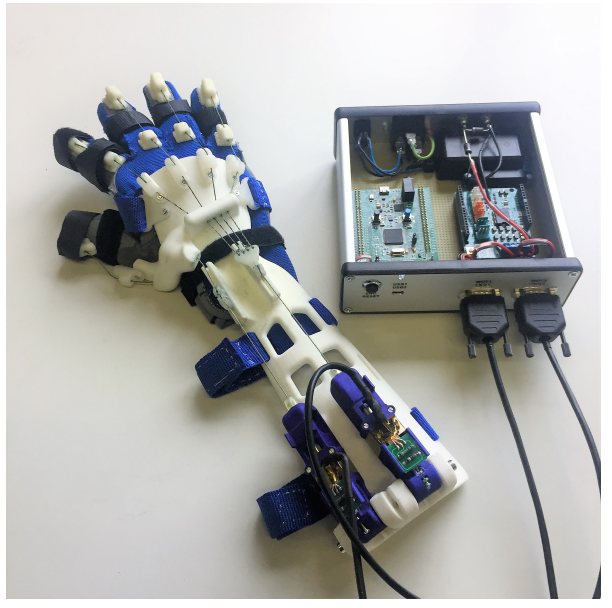

Fig. 7. ExoTen-Glove and Controller box.

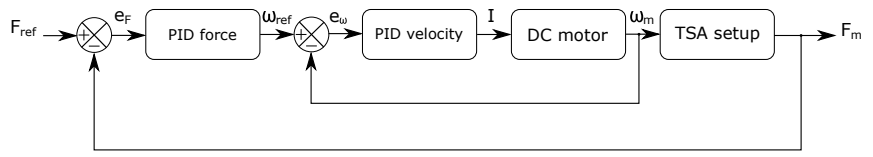

Fig. 8. Block diagram of the TSA's force and velocity control loop. Subscripts $r e f$ and $m$ denotes reference and measured, respectively. Meanwhile $F, \omega, I$ and $e$ represents force, velocity, current, and error respectively.

vii) a linear guide; on the main frame to prevent the twisting of the strings itself and with a separator to guide the tendons trough each finger.

In order to adjust the length of tendons for each individual user, we can twist the tendon on each individual finger.

\section{F. Electronics}

A STM32F4 32-bit microcontroller and a L298-based Arduino motor driver consists the electronic units (see Fig. 7). The design choice was made by considering the performance of the overall system while keeping the cost affordable. 


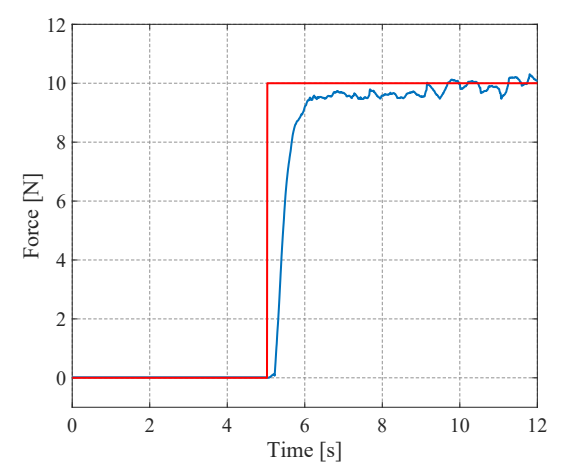

Fig. 9. The step response of the TSA force controller. The control bandwidth is approximately $2 \mathrm{rad} / \mathrm{s}$.

The microcontroller performs signal processing and control algorithm calculation including the ADC measurements as well as velocity and force control. Communication between the microcontroller and the PC is established through a serial USB communication.

\section{G. Controller}

A cascaded PID controller with inner velocity control loop and outer force control loop was implemented. Figure 8 shows the block diagram of the overall control scheme. The outer loop runs with a slower frequency of $1 \mathrm{kHz}$ while the inner loop is executed with $5 \mathrm{kHz}$ frequency. Since the force control response is greatly affected by the force sensor noise, we filter the measured force signal in order to obtain a smoother response. A first order low pass filter is used whose parameter is selected such that the trade-off between delay and noise level is balanced.

We tune the PID control gains by observing the dynamic response with respect to step and sinusoidal reference signal. A time constant of at least 0.5 second is desired to obtain a satisfactory force tracking. Figure 9 shows the controller step response. The rise time indicates that the time constant requirement is satisfied without overshoot. However, there is still an oscillatory behavior exhibited during the steady state. This is mostly due to the force sensor noise which is not filtered out entirely. Nevertheless, during the qualitative check, this noise does not affect much the realistic feeling of the ExoTen-Glove. As for the sinusoidal reference, the force tracking response and error are shown in Figure 10. The response yields a phase delay of about 0.3 second which is acceptable for our application.

Once the desired dynamic response for the force controller is obtained, the virtual stiffness emulation is then performed based on the measured displacement of the string. We tested various virtual stiffness and validate the measured force. Figure 11 shows the measured force during experiments with 2 different stiffness values: $2 \mathrm{~N} / \mathrm{mm}$ and $8 \mathrm{~N} / \mathrm{mm}$. As evident from the plot, the measured force matches the desired force curve and is proportional to the displacement, verifying the virtual stiffness emulation.

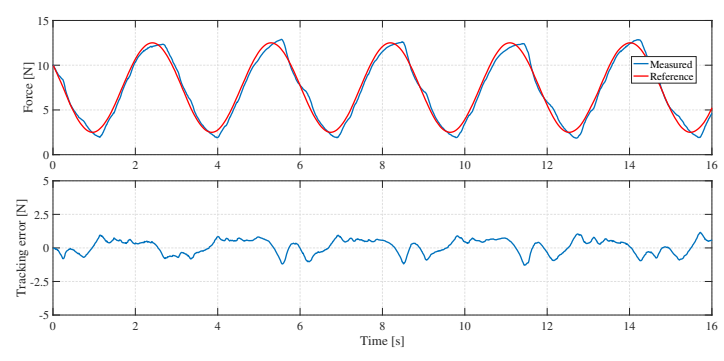

Fig. 10. The force control response given in a sine reference with a frequency of $0.3 \mathrm{~Hz}$.

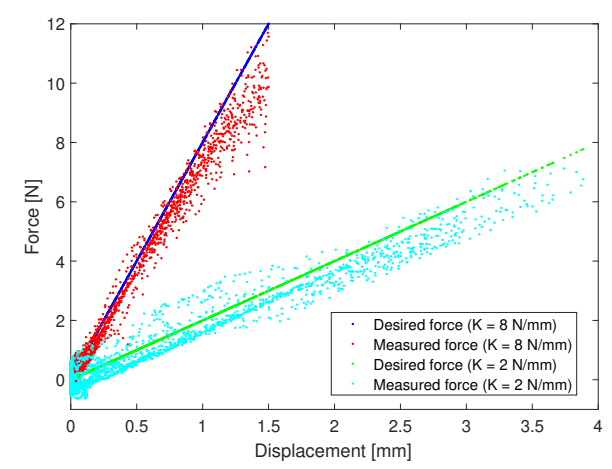

Fig. 11. The desired and measured force with respect to the deformation/displacement for two different stiffness values.

\section{EXPERIMENTAL EVALUATION}

A TSA based haptic glove is used in this experiment. It is lightweight system that tracks each finger movements in 1-DOF and renders high, stable grasping force to enable transparent interactions with virtual reality. The participants are asked to seat on a chair and wear the ExoTen-Glove and HTC VIVE headset while finger movements and interactions with virtual objects were presented on a HTC VIVE system (see Fig. 12). A desktop PC (Intel(R) Xeon(R) $2.40 \mathrm{GHz}$ $2.39 \mathrm{GHz}$ CPU (2 processors), $24 \mathrm{~GB}$ of RAM and with NVIDIA GeForce GTX 1060 3GB Graphic card) has been used for the experiment. CHAI3D that is an open source platform is used for modeling and simulating the haptics and development of a virtual world (with a virtual hand and spring). In order to make CHAI3D to support ExoTen-Glove, its libraries are extended by adding the drivers and libraries of ExoTen-Glove.

\section{A. Subjects}

In this experiment 8 healthy, male (7 right-handed and 1 left-handed) participants took part with an average age of 31 years (ranging from 26 to 36 years old) with normal or corrected to normal vision. Each experiment took around 20 minutes. Participants were informed about the research goal of the experiment and could ask questions and comment on the research after the experiment. 
TABLE I

CHARACTERISTICS OF SOME CURRENT HAPTIC INTERFACES

\begin{tabular}{cccccccc}
\hline \hline Device name & Actuation Type & Motion Type & Range of Motion & Fingers & Force Feedback & Max. Force & Total Weight \\
\hline \hline CyberGrasp [29] & DC motor/Cable driven & bidirectional & Full Hand Closure & 5 & Variable Stiffness & $12 \mathrm{~N}$ & $450 \mathrm{grams}$ \\
DEXMO [31] & Micro Servo Unit & bidirectional & Full Hand Closure & 5 & Variable Stiffness & $29.4 \mathrm{~N}$ & $320 \mathrm{grams}$ \\
Wolverine [32] & One way brake & unidirectional & $20-160 \mathrm{~mm}$ & 3 & Constant Stiffness & $106 \mathrm{~N}$ & $55 \mathrm{grams}$ \\
Rutger Master II [30] & Pneumatic Cylinder & unidirectional & $27 \mathrm{~mm}$ Stroke & 4 & Variable Stiffness & $16 \mathrm{~N}$ & $185 \mathrm{grams}$ \\
DESR [33] & Dielectric Elastomer & unidirectional & $5 \mathrm{~mm}$ Stroke & 4 & Variable Stiffness & $7.2 \mathrm{~N}$ & $38 \mathrm{grams}$ \\
ExoTen-Glove & TSA system & unidirectional & Full Hand Closure & 5 & Variable Stiffness & $80 \mathrm{~N}$ & $360 \mathrm{grams}$ \\
\hline
\end{tabular}

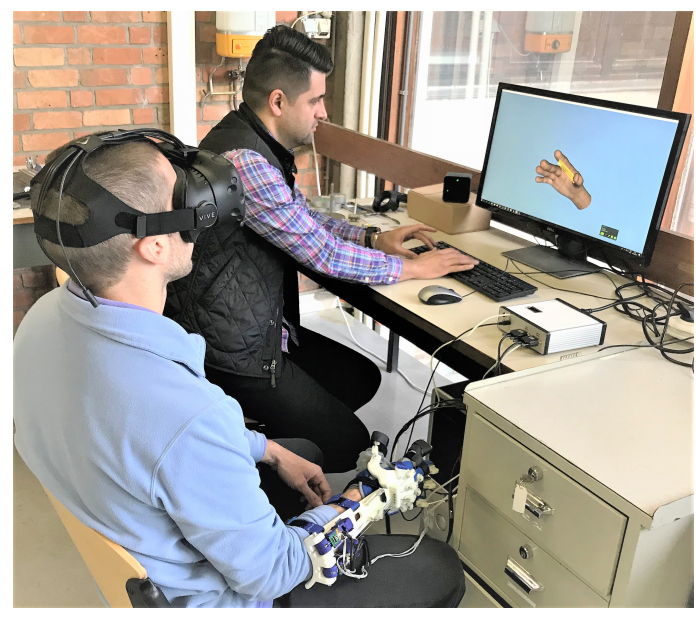

Fig. 12. The experimental setup: In this experiment participants were asked to wear the HTC VIVE headset and ExoTen-Glove and squeeze a set of virtual springs with different stiffness by their thumb and index finger.

\section{B. Experiment Procedure}

Participants were informed to squeeze a real spring (with the stiffness of $2 \mathrm{~N} / \mathrm{mm}$ ) with their thumb and index finger before trying out the virtual springs. They were asked to become familiar with the stiffness value of the real spring and keep in mind while trying out the virtual springs. They wore the ExoTen-Glove and HTC VIVE headset (see Fig. 12). Virtual springs with two different stiffness $(2 \mathrm{~N} / \mathrm{mm}$ and $8 \mathrm{~N} / \mathrm{mm}$ ) one with visually same to the real spring and the other one visually different were presented. Each stiffness value is presented 5 times in random order.

To enable the virtual spring to be compressed the same amount, the index finger started from a fully open position to a predefined closed position. Participants presented with a virtual spring and allowed to squeeze it until they are sure about their answer. Their responses response time, applied force and positions are recorded.

\section{Analysis and Results}

The accuracy of stiffness experiment results is presented in Figure 13. The accuracy is calculated as the percentage of correct answers of matching spring difference with the real spring value. White bars represent the conditions in which the stiffness value presented in ExoTen-Glove is visually same presented at HTC VIVE headset. Grey bars represent the trials in which the stiffness value presented in ExoTen-Glove is visually different presented at the HTC VIVE headset. The bars on the left side are for the stiffness value same to real spring $(2 \mathrm{~N} / \mathrm{mm})$ and the bars on the right side are for the stiffness value different to real spring $(8 \mathrm{~N} / \mathrm{mm})$. Lines on the bars show the standard errors. The figure shows that the overall accuracy of the participants detecting spring stiffness with real spring is $77.5 \%$. Participants have highest accuracy in detecting the spring stiffness difference with the real spring when the stiffness is different at the ExoTenGlove and visually different at the HTC VIVE headset. The lowest accuracy in detecting the spring stiffness difference with the real spring is when the stiffness value at the ExoTenGlove is same with the real spring and visually different at the HTC VIVE headset.

T-test is performed for the analysis of the accuracy of both springs. Our results show that virtual spring with the stiffness of $8 \mathrm{~N} / \mathrm{mm}$ had a significantly higher accuracy in comparing the stiffness difference compared to spring with the stiffness of $2 \mathrm{~N} / \mathrm{mm}((\mathrm{t}(7)=5.11 ; \mathrm{p}<0.001))$. Subjects have about a $90 \%$ accuracy of detecting the stiffer spring as different to real spring. The analysis of reaction times does not result in any significant difference $((\mathrm{t}(7)=0.6 ; \mathrm{p}=0.56))$.

\section{Discussion}

In this study, evaluation of the compact and light-weight wearable haptic glove (ExoTen-Glove) was presented. The system was evaluated with an experiment in which participants compared the stiffness value of a real spring while interacting with virtual reality. In this experiment, the subjects squeezed two different virtual springs with their thumb and index finger. One of the spring was visually same with the real spring and the other one was visually different. More specifically one of the spring looked to have same stiffness visually, one looked visually different stiffness with the real spring. The aim was to find out how well they could differentiate the stiffness differences of two springs with a TSA-based haptic glove when they were presented visually same and visually different. We expected that the users would have difficulty in distinguishing when the stiffness is same at the ExoTen-Glove with real spring and visually different at the HTC VIVE headset. Our result confirmed our hypothesis. 


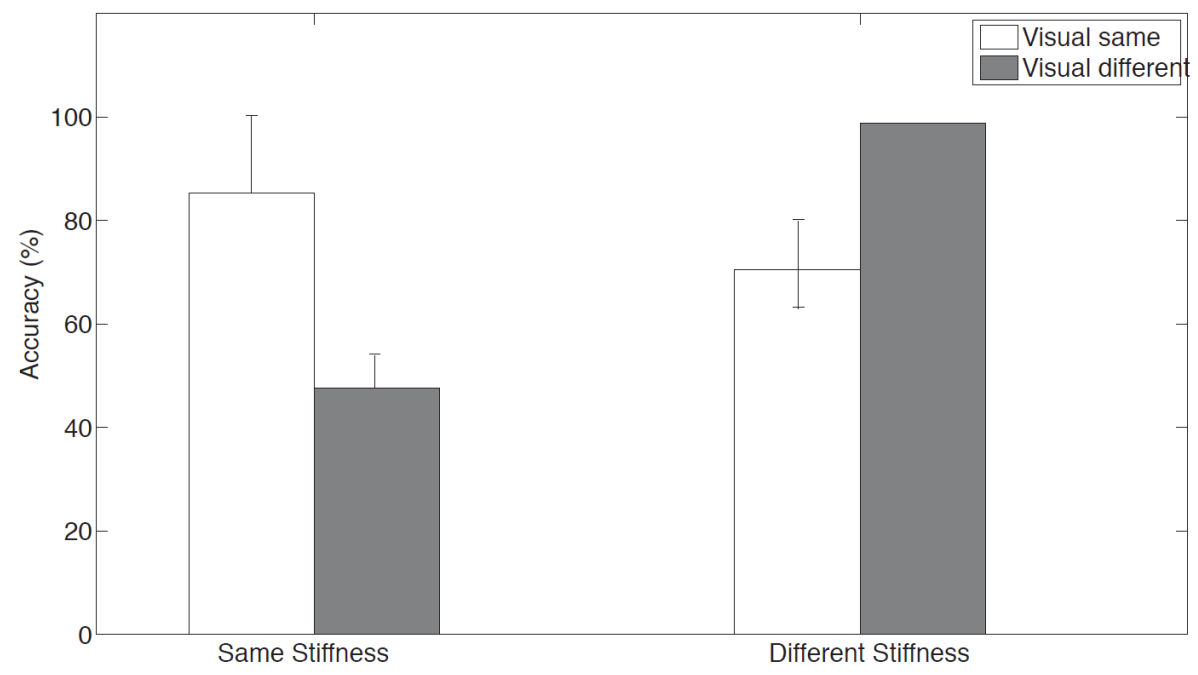

Fig. 13. Experimental results: Percentage of correct answers of stiffness differences with error bars

Users had highest difficulties in detecting spring difference with the real spring when the stiffness value was same at the ExoTen-Glove while it was presented visually different. The result of the present study illustrated that users use not only haptic cues but also visual cues in detecting spring stiffness difference.

\section{Comparison of the Existing Devices}

Table I presents a comparison charts of the existing haptic devices; CyberGrasp [29], Rutgers Master II-ND glove [30], DEXMO [31], Wolverine [32] and DESR [33] that has been developed in the recent decade.

The ExoTen-Glove can provide $80 N$ force, from each actuator, which is suitable to human finger capability. According to the previous study [39], the average strength of the chuck pinch is $77.4 N$. It is worth mentioning that since the TSA system has a very high transmission ratio, the force range and therefore the complaint frame design of the force sensor can be adjusted in case the particular application is needed.

\section{CONCLUSIONS}

Designing a wearable haptic device that is compact enough to be worn and at the same time powerful enough to provide stable force feedback is a challenging task. In this paper a compact wearable haptic glove based on TSA system has been presented. The TSA is a simple, lightweight and lowcost actuation system. Hence it is suitable for wearable haptic devices.

An evaluation of the proposed haptic glove is done with an experiment. Users compared the stiffness value of a real spring with a pair of virtual springs. The aim of the study is to illustrate the benefit of using TSA system in distinguishing stiffness of a pair of virtual springs and the role of the visual feedback. Users were able to identify the spring stiffness difference better when the haptic feedback from the ExoTenGlove and visual feedback presented at the HTC VIVE Headset are different with the real spring.

Cost-effective haptic glove has many benefits for the upper limb rehabilitation. The VR environment coupled with games and robotics allows customized, repetitive and effective rehabilitation for each user. VR application merged with haptics would also increase the efficiency of the rehabilitation of stroke patients. Currently, we are working on the next version of the haptic glove. In order to reduce its weight and increase the realism of haptic feedback, we will add composite linkages. Additionally, we are integrating Unity game engine into CHAI3D platform for complex hand animations. In the future, the haptic glove will be extended for more complex grasping tasks by adding independent actuators for each finger and controlling them separately.

\section{REFERENCES}

[1] A. Bardorfer, M. Munih, A. Zupan, and A. Primozic, "Upper limb motion analysis using haptic interface," IEEE/ASME Trans. on Mechatronics, vol. 6, no. 3, pp. 253-260, 2001.

[2] C. Basdogan, C. H. Ho, and M. A. Srinivasan, "Virtual environments for medical training: graphical and haptic simulation of laparoscopic common bile duct exploration," IEEE/ASME Transactions on Mechatronics, vol. 6, no. 3, pp. 269-285, 2001.

[3] I. Ivanisevic and V. J. Lumelsky, "Configuration space as a means for augmenting human performance in teleoperation tasks," Trans. Sys. Man Cyber. Part B, vol. 30, no. 3, pp. 471-484, 2000.

[4] R. V. Dubey, S. E. Everett, N. Pernalete, and K. A. Manocha, "Teleoperation assistance through variable velocity mapping," IEEE Trans. on Robotics and Automation, vol. 17, no. 5, pp. 761-766, 2001.

[5] M. Guthold, M. R. Falvo, W. G. Matthews, S. Paulson, S. Washburn, D. A. Erie, R. Superfine, F. P. Brooks, and R. M. Taylor, "Controlled manipulation of molecular samples with the nanomanipulator," IEEE/ASME Trans. on Mechatronics, vol. 5, no. 2, pp. 189-198, 2000.

[6] S. Marlière, D. Urma, J. Florens, and F. Marchi, "Multi-sensorial interaction with a nano-scale phenomenon: the force curve," in Proc. of EuroHaptics, 2004, pp. 246-253.

[7] S. W. Lee, K. A. Landers, and H.-S. Park, "Development of a biomimetic hand exotendon device (biomhed) for restoration of functional hand movement post-stroke," IEEE Trans. on Neural Systems and Rehabilitation Engineering, vol. 22, no. 4, pp. 886-898, 2014. 
[8] A. Battezzato, "Towards an underactuated finger exoskeleton: An optimization process of a two-phalange device based on kinetostatic analysis," Mechanism and Machine Theory, vol. 78, pp. 116 - 130, 2014.

[9] — "Kinetostatic analysis and design optimization of an n-finger underactuated hand exoskeleton," Mechanism and Machine Theory, vol. 88, pp. 86-104, 2015.

[10] J. Iqbal, H. Khan, N. G. Tsagarakis, and D. G. Caldwell, "A novel exoskeleton robotic system for hand rehabilitation - conceptualization to prototyping," Biocybernetics and Biomedical Engineering, vol. 34, no. 2, pp. $79-89,2014$.

[11] H. Kawasaki, Y. Doi, S. Koide, T. Endo, and T. Mouri, "Hand haptic interface incorporating 1d finger pad and 3d fingertip force display devices," in Proc. Int. Symposium on Industrial Electronics, 2010, pp. 1869-1874.

[12] H. Kawasaki and T. Mouri, "Design and control of five-fingered haptic interface opposite to human hand," IEEE Trans. on Robotics, vol. 23, no. 5, pp. 909-918, 2007.

[13] S. S. Fisher, E. M. Wenzel, C. Coler, and M. W. McGreevy, "Virtual interface environment workstations," in Proceedings of the Human Factors Society Annual Meeting, vol. 32, no. 2. SAGE Publications Sage CA: Los Angeles, CA, 1988, pp. 91-95.

[14] H. Kawasaki, "Force feedback glove for manipulation of virtual objects," Jour. Of Robotics and Mechatronics, vol. 5, no. 1, pp. 79-84, 1993.

[15] C. A. Avizzano, F. Bargagli, A. Frisoli, and M. Bergamasco, "The hand force feedback: analysis and control of a haptic device for the humanhand," in Systems, Man, and Cybernetics, 2000 IEEE International Conference on, vol. 2, 2000, pp. 989-994 vol.2.

[16] M. Achibet, G. Casiez, and M. Marchal, "DesktopGlove: a Multifinger Force Feedback Interface Separating Degrees of Freedom Between Hands," in 3DUI'16, the 11th Symposium on 3D User Interfaces, ser. Proc. of the Symposium on 3D User Interfaces, I. C. Society, Ed., Greenville, United States, 2016, p. 10.

[17] M. Fontana, A. Dettori, F. Salsedo, and M. Bergamasco, "Mechanical design of a novel hand exoskeleton for accurate force displaying," in Robotics and Automation, 2009. ICRA'09. IEEE International Conference on. IEEE, 2009, pp. 1704-1709.

[18] P. Galambos, "Vibrotactile feedback for haptics and telemanipulation: Survey, concept and experiment," Acta Polytechnica Hungarica, vol. 9, no. 1, pp. 41-65, 2012.

[19] R. Montaño-Murillo, R. Posada-Gómez, A. Martínez-Sibaja, B. Gonzalez-Sanchez, A. Aguilar-Lasserre, and P. Cornelio-Martínez, "Design and assessment of a remote vibrotactile biofeedback system for neuromotor rehabilitation using active markers," Procedia Technology, vol. 7, pp. 96 - 102, 2013.

[20] P. Olsson, S. Johansson, F. Nysjö, and I. Carlbom, "Rendering stiffness with a prototype haptic glove actuated by an integrated piezoelectric motor," in Proc. Int. Conf. on Haptics: Perception, Devices, Mobility, and Communication, ser. EuroHaptics'12, 2012, pp. 361-372.

[21] C. Tejeiro, C. E. Stepp, M. Malhotra, E. Rombokas, and Y. Matsuoka, "Comparison of remote pressure and vibrotactile feedback for prosthetic hand control," in 2012 4th IEEE RAS EMBS International Conference on Biomedical Robotics and Biomechatronics (BioRob), 2012, pp. 521-525.

[22] G. A. Tabot, J. F. Dammann, J. A. Berg, F. V. Tenore, J. L. Boback, R. J. Vogelstein, and S. J. Bensmaia, "Restoring the sense of touch with a prosthetic hand through a brain interface," Proceedings of the National Academy of Sciences, vol. 110, no. 45, pp. 18279-18 284, 2013.

[23] G. Palli, C. Natale, C. May, C. Melchiorri, and T. Würtz, "Modeling and control of the twisted string actuation system," IEEE/ASME Trans. on Mechatronics, vol. 18, no. 2, pp. 664-673, 2013.

[24] S. Moshe, "Twisting wire actuator," Journal of Mechanical Design, vol. 127, no. 3, p. 441-445, jul 2004.

[25] G. Palli, C. Melchiorri, G. Vassura, U. Scarcia, L. Moriello, G. Berselli, A. Cavallo, G. De Maria, C. Natale, S. Pirozzi, C. May, F. Ficuciello, and B. Siciliano, "The DEXMART Hand: Mechatronic design and experimental evaluation of synergy-based control for human-like grasping," The Int. Journal of Robotics Research, vol. 33, no. 5, pp. 799-824, 2014.

[26] D. Popov, I. Gaponov, and J. Ryu, "A preliminary study on a twisted strings-based elbow exoskeleton," in World Haptics Conference (WHC), 2013. IEEE, 2013, pp. 479-484.
[27] A. Pepe, M. Hosseini, U. Scarcia, G. Palli, and C. Melchiorri, "Development of an haptic interface based on twisted string actuators," in Advanced Intelligent Mechatronics (AIM), 2017 IEEE International Conference on. IEEE, 2017, pp. 28-33.

[28] T. H. Massie, J. K. Salisbury, et al., "The phantom haptic interface: A device for probing virtual objects," in Proceedings of the ASME winter annual meeting, symposium on haptic interfaces for virtual environment and teleoperator systems, vol. 55, no. 1. Citeseer, 1994, pp. 295-300.

[29] "Cybergrasp," http://www.cyberglovesystems.com , accessed: 201804-01.

[30] M. Bouzit, G. Burdea, G. Popescu, and R. Boian, "The rutgers master ii-new design force-feedback glove," IEEE/ASME Transactions on mechatronics, vol. 7, no. 2, pp. 256-263, 2002.

[31] X. Gu, Y. Zhang, W. Sun, Y. Bian, D. Zhou, and P. O. Kristensson, "Dexmo: An inexpensive and lightweight mechanical exoskeleton for motion capture and force feedback in vr," in Proceedings of the 2016 CHI Conference on Human Factors in Computing Systems, ser. CHI '16. New York, NY, USA: ACM, 2016, pp. 1991-1995. [Online]. Available: http://doi.acm.org/10.1145/2858036.2858487

[32] I. Choi, E. W. Hawkes, D. L. Christensen, C. J. Ploch, and S. Follmer, "Wolverine: A wearable haptic interface for grasping in virtual reality," in Intelligent Robots and Systems (IROS), 2016 IEEE/RSJ International Conference on. IEEE, 2016, pp. 986-993.

[33] R. Zhang, A. Kunz, P. Lochmatter, and G. Kovacs, "Dielectric elastomer spring roll actuators for a portable force feedback device," in Haptic Interfaces for Virtual Environment and Teleoperator Systems, 2006 14th Symposium on. IEEE, 2006, pp. 347-353.

[34] T. Würtz, C. May, B. Holz, C. Natale, G. Palli, and C. Melchiorri, "The twisted string actuation system: Modeling and control," in Advanced Intelligent Mechatronics (AIM), 2010 IEEE/ASME International Conference on. IEEE, 2010, pp. 1215-1220.

[35] M. Hosseini, G. Palli, and C. Melchiorri, "Design and implementation of a simple and low-cost optoelectronic force sensor for robotic applications," in Advanced Intelligent Mechatronics (AIM), 2016 IEEE International Conference on. IEEE, 2016, pp. 1011-1016.

[36] G. Palli, M. Hosseini, and C. Melchiorri, "A simple and easy-to-build optoelectronics force sensor based on light fork: Design comparison and experimental evaluation," Sensors and Actuators A: Physical, vol. 269, pp. 369-381, 2018.

[37] M. Hosseini, R. Meattini, G. Palli, and C. Melchiorri, "A wearable robotic device based on twisted string actuation for rehabilitation and assistive applications," Journal of Robotics, vol. 2017, 2017.

[38] — , "Development of semg-driven assistive devices based on twisted string actuation," in Control, Automation and Robotics (ICCAR), 2017 3rd International Conference on. IEEE, 2017, pp. 115-120.

[39] A. B. Swanson, I. B. Matev, and G. De Groot, "The strength of the hand," Bull Prosthet Res, vol. 10, no. 14, pp. 145-153, 1970. 